\title{
Electrophorèse de I'ADN en champ pulsé
}

La technique d'électrophorèse en gel d'agarose ou de polyacrylamide permet de séparer des molécules d'ADN suivant leur taille à condition qu'elle n'excède pas 50000 paires de bases (50 kilobases ou kb). Les gels d'agarose ou d'acrylamide sont constitués d'un réseau désorganisé de longues fibres baigné dans un milieu aqueux. On place les molécules d'ADN qu'on désire séparer en un point du gel et on applique un champ électrique. Sous son action, les molécules d'ADN chargées négativement se déplacent à travers le réseau. On constate expérimentalement que plus elles sont petites, plus elles se déplacent rapidement. Ainsi peut-on les séparer. Cette séparation est due aux fibres du réseau, car, en leur absence, les molécules d'ADN se déplacent toutes avec la même vitesse quelle que soit leur taille. Cependant l'électrophorèse perd tout pouvoir résolutif pour des molécules d'ADN de taille supérieure à $50 \mathrm{~kb}$; par exemple, l'ADN du bactériophage lambda (50 kb) et l'ADN des chromosomes de la levure (de 300 à $2000 \mathrm{~kb}$ ) migrent à peu près à la même vitesse (figure 1). En d'autres termes, au-delà de $50 \mathrm{~kb}$, la mobilité électrophorétique de l'ADN ne dépend plus de sa longueur. En 1984, deux chercheurs de l'université de Columbia à New York, Schwartz et Cantor [1], proposent une méthode d'électrophorèse sur gel d'agarose dont le pouvoir de séparation peut
[9]. Comment cela est-il possible ? Commençons par quelques ordres de grandeur. Les distances moyennes qui séparent des fibres voisines dans ces gels sont de l'ordre de 150 à 400 nanomètres (nm) $\left(1 \mathrm{~nm}=10^{-9} \mathrm{~m}\right)$. La flexibilité des molécules d'ADN peut être appréciée en mesurant la distance minimale devant séparer deux points $A$ et $B$ quelconques de cette molécule pour que son orientation au point A n'ait pas d'influence sur son orientation au point B. Cette distance, appelée longueur de persistance, est de l'ordre de $150 \mathrm{~nm}$. Entre deux fibres du gel, l'ADN peut donc tout juste faire une boucle.

Une molécule d'ADN de $50 \mathrm{~kb}$ possède une longueur totale de $18000 \mathrm{~nm}$ et, dans le gel, en l'absence de champ électrique, elle apparaît comme une longue chaîne serpentant au hasard entre les fibres du gel : une sorte de pelote statistique dans un réseau enchevêtré (figure 2). La distance moyenne entre deux fibres étant comparable à la longueur de persistance, la chaîne est complètement contrainte et, seules les deux extrémités ont suffisamment de degrés de liberté pour avancer. Une des deux extrémités détermine le chemin et le reste de la molécule coulisse entre les fibres comme un serpent dans un jeu de quilles. En l'absence de champ électrique, le sens du déplacement est indifféremment déterminé par l'une ou l'autre des deux extrémités et peut s'effectuer dans toutes les orientations possibles. $\mathrm{Ce}$ mou- vement de la chaîne appelé mouvement de reptation [2] est un coulissement selon un chemin parcouru au hasard.

Sous l'influence d'un champ électrique, la chaîne chargée est soumise à une force électrostatique. Elle se déplace toujours par reptation mais, cette fois-ci, l'une des deux extrémités au lieu de prendre une direction au hasard va se déplacer préférentiellement dans celle imposée par le champ électrique [3-5]. On devine l'effet produit par une telle dynamique : l'enveloppe de la molécule d'ADN qui était sphérique en l'absence de champ (pelote statistique) va s'allonger dans le sens du champ électrique sous son influence ; en d'autres termes, le serpent se déplie puisque sa tête se déplace dans une direction déterminée (figure 2). Cette déformation est un effet du gel car, en son absence, il faudrait appliquer des champs 100 fois plus forts pour l'observer. L'expression de la mobilité électrophorétique de ces longues molécules d'ADN est la somme de deux termes $m_{O}+m_{E}$ qui se comprennent comme les deux premiers termes d'un développement selon les puissances du champ électrique : le premier terme $\mathrm{m}_{\mathrm{O}}$ qui ne dépend pas du champ électrique (mobilité à champ électrique faible) correspond au déplacement de l'ADN non déformé. Un calcul [7, 8] montre qu'il varie comme l'inverse de la longueur de la molécule. Le second terme $\mathrm{m}_{\mathrm{E}}$ dépend du carré du champ électrique et rend compte 


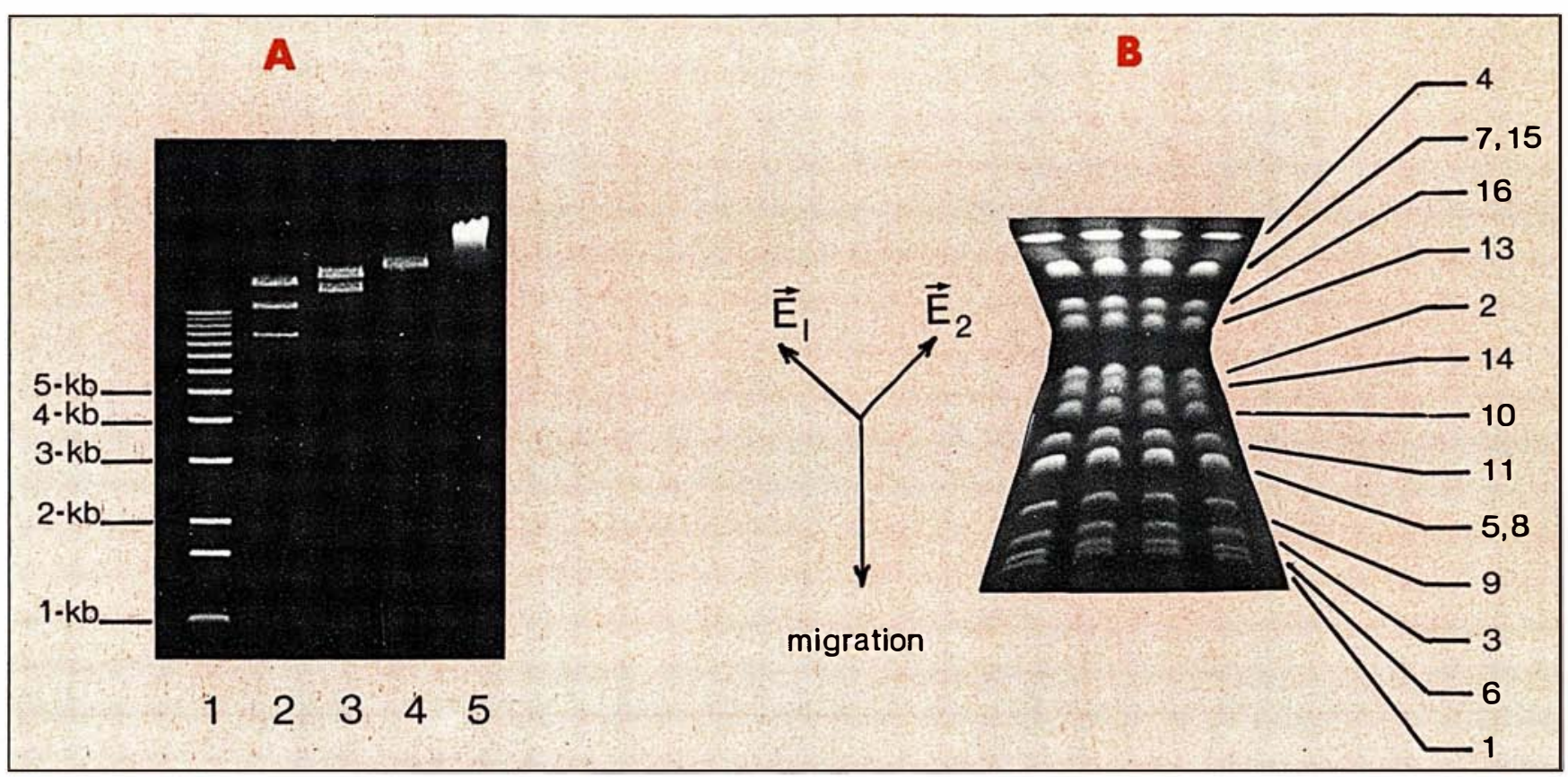

Figure 1. A. Migration électrophorétique en champ électrique constant de molécules d'ADN de longueur variable. La migration s'effectue de haut en bas. 1 = fragments d'ADN obtenus par polymérisation d'un fragment de $1 \mathrm{~kb}$. Le fragment le plus long est de $12 \mathrm{~kb} ; 2$ = trois fragments de restriction de I'ADN du bactériophage lambda de longueur $22 \mathrm{~kb}, 14 \mathrm{~kb}$ et $9 \mathrm{~kb} ; 3=$ deux fragments de restriction de l'ADN du bactériophage de longueur $30 \mathrm{~kb}$ et $17 \mathrm{~kb}$. 4 : L'ADN du bactériophage lambda, 48-kb. 5 : L'ADN total de la levure Saccharomyces cerevisiae. Bien que les tailles des ADN chromosomiques soient comprises entre $300 \mathrm{~kb}$ et $2000 \mathrm{~kb}$ (voir B de cette même figure), leurs migrations électrophorétiques sont identiques et très voisines de celles de l'ADN du bactériophage lambda. B. Migration électrophorétique on champs électriques alternatifs. Cette méthode, analogue à celle en champs pulsés (voir texte), consiste à appliquer alternativement deux champs électriques suivant des directions orthogonales : $E_{1}$ et $E_{2}$. La direction de migration globale se fera donc suivant la bissectrice de l'angle. Les numéros de chaque chromosome sont indiqués sur la droite. Deux chromosomes sont absents : le chromosome 17 qu'à ce jour personne n'a encore pu identifier et le chromosome 12. Ce dernier, le plus grand de tous, ne migre pas dans les conditions utilisées ici. Deux paires de chromosomes $(5,8)$ et $(7,15)$ ont des longueurs voisines et donnent naissance à deux doublets. On peut les résoudre en utilisant d'autres souches de levure, cet organisme présentant un polymorphisme des longueurs de ses ADN chromosomiques.

de la conformation allongée prise par la molécule soumise à son effet. Il est indépendant de la longueur de la molécule. Pour des molécules supérieures à $50 \mathrm{~kb}$, et avec des champs électriques utilisables en pratique, le premier terme est petit devant le second et la mobilité est pratiquement indépendante de la longueur. Ceci explique la perte de résolution observée. Comment l'éviter ? L'idée de Schwartz et Cantor est fort simple. Au moment où l'on applique le champ électrique l'enveloppe de la molécule d'ADN $\mathrm{m} / \mathrm{s} n^{\circ} 1$ vol. 4, janvier 88 commence à se déformer par son déplacement. Le temps mis par une molécule d'ADN pour s'allonger dans le sens du champ, qui est aussi le temps de passage de la forme à enveloppe sphérique à celle à enveloppe oblongue (figure 2) et qu'on appelle le temps caractéristique d'allongement, est à peu près égal au temps nécessaire à cette molécule pour se déplacer d'une distance égale à sa longueur: il dépend donc de sa longueur. $\mathrm{Au}$ moment où l'on applique le champ électrique, la mobilité est $\mathrm{m}_{\mathrm{O}}$. Elle augmente progressivement pour atteindre au bout du temps caractéristique d'allongement la valeur $\mathrm{m}_{\mathrm{O}}+$ $\mathrm{m}_{\mathrm{E}}$. Considérons maintenant deux molécules d'ADN de longueur différente et appliquons le champ électrique pendant un temps compris entre les deux temps caractéristiques d'allongement. Seule la plus petite aura la possibilité de s'allonger suffisamment pour atteindre la mobilité maximale. Pour pouvoir exploiter cette différence transitoire de mobilité, il suffit alors d'arrêter le champ pendant un temps suffisant 


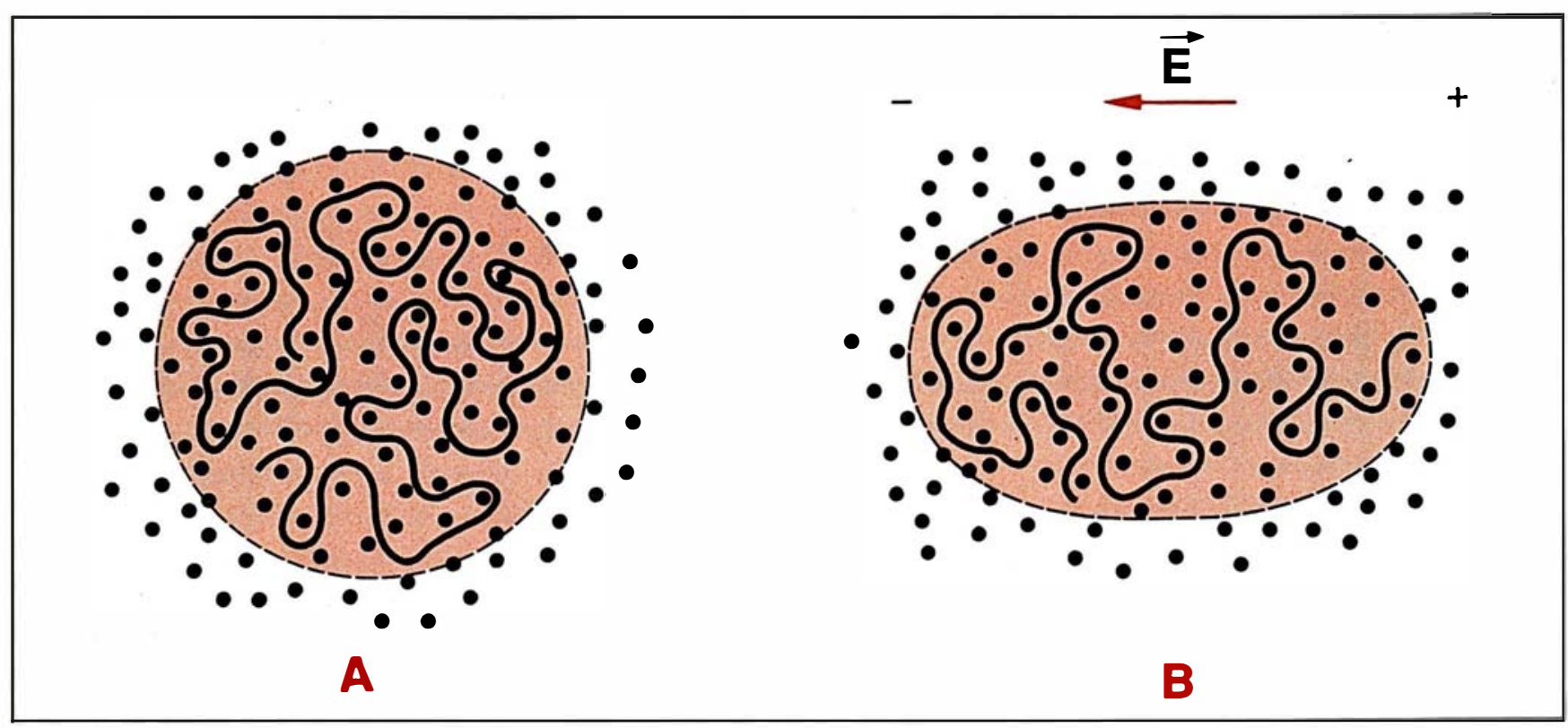

Figure 2. Représentation schématique à deux dimensions d'une molécule d'ADN dans un gel d'agarose. Les fibres représentées par des points sont distribuées au hasard; I'ADN serpente entre elles, la courbure de l'ADN et la distance moyenne entre deux fibres voisines étant approximativement identiques (voir texte). A : En absence de champ électrique, l'enveloppe de la molécule d'ADN marquée en pointillés est isotrope (sphérique). B : Sous l'influence d'un champ électrique $\vec{E}$ indiqué par une flèche rouge horizontale allant de droite à gauche, la molécule se déplace vers la droite en même temps qu'elle se déforme : son enveloppe s'allonge dans le sens du champ électrique.

pour que les molécules reviennent à leur forme de repos puis de recommencer ce cycle un grand nombre de fois. On peut également, au lieu d'arrêter le champ électrique, changer son orientation et jouer ainsi sur le temps mis par la molécule d'ADN pour s'allonger dans la nouvelle direction : en appliquant alternativement des champs électriques dans des directions orthogonales $[1,6,9]$, on obtient des séparations équivalentes à celles observées avec la méthode en champ pulsé. Un exemple en est donné dans la figure 1 .

Pour la biologie moléculaire, cette nouvelle technique confine au sensationnel. Grâce à elle, il est pratiquement possible, pour tous les organismes à petit génome, procaryotes et eucaryotes inférieurs, de localiser très rapidement un gène quelconque. Par exemple, 16 des 17 chromosomes de la levure Saccharomyces cerevisiae peuvent être champs pulsés (figure 1) et ont été identifiés par hybridation avec des gènes clonés dont on connaissait la localisation chromosomique. On peut ainsi en une journée trouver le (ou les) chromosome(s) portant un gène qu'on vient de cloner et il est même possible par une procédure simple de déterminer sa position par rapport au centromère. Les méthodes génétiques précédemment utilisées demandaient des semaines pour obtenir une telle information avec une précision bien moindre. L'ADN correspondant à un chromosome peut aussi être sorti du gel et utilisé pour faire une banque génomique restreinte. En ordonnant, par des procédés informatiques simples, les différents fragments de cette banque, on peut reconstituer la carte de l'ADN du chromosome entier.

Certains ADN chromosomiques ne peuvent pas, pour l'instant, être séparés par cette technique : il s'agit d'ADN linéaires dont la longueur dépasse $9000 \mathrm{~kb}$ (l'ADN des chromosomes d'eucaryotes supérieurs) ou des molécules d'ADN circulaires comme par exemple le génome d'E. coli $(5000 \mathrm{~kb})$. On peut tout de même aborder leur étude en les coupant par des enzymes de restriction dont les sites de reconnaissance sont rares. Deux enzymes de restriction commercialisées, Notl et Sfil, reconnaissent des sites à 8 bases, alors que la plupart des autres enzymes reconnaissent des sites ayant, au plus, six bases. Les fragments créés par ces enzymes sont des molécules linéaires de taille inférieure, en général, à $2000 \mathrm{~kb}$. Ils peuvent alors être étudiés par électrophorèse en champ électrique pulsé. Les résultats de telles expériences permettent de situer les fragments les uns par rapport aux autres et conduisent à la construction de cartes génétiques locales extrêmement précises pouvant s'étendre sur plusieurs millions de paires de 
bases. Plusieurs régions du génome humain ont fait l'objet de telles études. Citons, par exemple, celle du gène responsable de la myopathie de Duchenne $[10,11]$ et la région HLA [12, 13].

Grâce à l'électrophorèse en champ électrique pulsé qui permet le fractionnement de molécules d'ADN de taille comprise entre 50 et $9000 \mathrm{~kb}$, un nouveau domaine de recherches en biologie moléculaire est en train de s'ouvrir

\section{François Caron}

Remerciements

Je tiens à remercier plus particulièrement H. Hervet et G. Thomas pour leur lecture approfondie de ce manuscrit et pour de judicieuses corrections.

\section{RÉFÉRENCES}

1. Schwartz DC, Cantor CR. Separation of yeast chromosome-sized DNAs by pulsed field gradient gel electrophoresis. Cell 1984; 37 67-75

2. De Gennes PG. Reptation of the polymer chain in the presence of fixed obstacles. $J$ Chem Phys $1971 ; 55$ : 572-8.

3. Lumpkin OJ, Déjardin $\mathrm{P}$, Zimm $\mathrm{BH}$ Theory of gel electrophoresis of DNA. Biopolymers $1985 ; 24: 1573-93$

4. Slater GW, Noolandi J. On the reptation theory of gel electrophoresis. Biopolymers 1986 ; $25: 431-54$.

5. Hervet H, Bean CP. Electrophoretic mobility of phage Hind III and Hae II DNA fragments in agarose gels : a detailed study. Bio polymers 1987 (sous presse)

6. Carle GF, Olson MV. Separation of chro mosomal DNA molecules from yeast by orthogonal-field-alternation gel electrophoresis Nucleic Acid Res 1984 ; 12 : 5647-64.

7. Lerman LS, Frisch HL. Why does the electrophoretic mobility of DNA in gels vary with the length of the molecule. Biopolymers 1982 ; 21: 995-7.
8. Lumpkin OJ, Zimm BH. Mobility of DNA in gel electrophoresis. Biopolymers $1982 ; 21$ : 2315-6.

9. Smith CL, Matsumoto T, Niwa O, et al. An electrophoretic karyotype for Schizosaccharomyces pombe by pulsed field gel electrophoresis. Nucleic Acids Res 1987 ; 15 : 4481-9.

10. Ommen GJB, Verkerk JMH, Hofker MH et al. A physical map of 4 million bp around the Duchenne muscular dystrophy gene on the human X-chromosome. Cell 1986; 47 : 499-504

11. Burmeister $M$, Lehrach $H$. Long-range restriction map around the Duchenne muscular dystrophy gene. Nature 1986 ; 324, 582-5.

12. Hardy DA, Bell JI, Long EO, Lindsten $T$, McDevitt HO. Mapping of the class II region of the human major histocompatibility complex by pulsed-field gel electrophoresis. Nature 1986 ; 323 : ‘453-5.

13. Lawrence SK, Smith CL, Srivasta R, Cantor CR, Weissman-SM. Megabase-scale mapping of the HLA gene complex by pulsed field gel electrophoresis. Science $1987 ; 235$ : 1387-90. 